\title{
Recognizing Face Image Based on Gabor and DCT Feature Extraction using SVM
}

\author{
Richa Khyalia ${ }^{1}$, Priyanka Trikha ${ }^{2}$ \\ Department of Computer Science \& Engineering, Swami Keshvanand Institute of Technology, Management \\ \& Gramothan, Jaipur-302017 (INDIA) \\ Email: richa.khyalia11@gmail.com1,trikhapriyanka@gmail.com \\ Received 16.01.2021, received in revised form 19.02.2021, accepted 19.02.2021
}

\begin{abstract}
This paper has proposed a hybrid approach by combining the Gabor filter and a Discrete Cosine Transform. Face recognition systems can use in authentication, human-computer interaction, surveillance, and lots of applications. These application users have demanded that a higher accuracy, more efficient, low-cost, and low-calculation time for the facial recognition system. The research issue in image recognition is to maximize recognition accuracy by improving the pre-processing of face-set images, developing the method of extraction of faces, as well as using the most effective face classifier. Feature extraction is an important step that can influence the accuracy of the recognition system. The advantage of the Gabor filter is that it may calculate a large number of features by projection in various directions and sizes. The problem with the Gabor filter is that it has a high dimension and high redundancy and that can be minimized by certain filtering and sampling techniques. In the proposed process, the Gabor features have been filtered by the sampling filtration and the Discrete Cosine Transform extracts the low-frequency features of the Gabor filter sampled. Then assign the obtained optimum features to the support vector machine (SVM) classifier. This algorithm aims to improve accuracy with fewer features for different expressions. The ORL (Olivetti Research Lab) face dataset has used for the experiment. The accuracy of the facial recognition method is 96 percent (verified by using 5 -fold crossvalidation).
\end{abstract}

Keywords- Face Recognition, Gabor Filter, Discrete Cosine Transform, and a Support Vector Machine classifier.

\section{INTRODUCTION}

Machine face recognition is growing as an active engineering field covering many fields such as image analysis, pattern recognition, machine vision, and neural networks. Face Recognition Technology has a range of industrial and law enforcement uses.

The human face has a major feature that can be seen as a person's identity. Computer-supported facial Recognition Technology builds up the image of a variety of human faces in the face database and recognizes the test image of a new person related to this database image and distinguishes individuals. This analysis approach is used in a wide variety of online applications [1]. The hybrid method is a perfect combination of multiple methods. The appearance-based approach appears to be more successful than the geometric-based approach because of the illumination noise the geometric approach has effected.

\section{REVIEW OF LITERATURE}

Zhao et. al. surveyed different faces in their paper, Recognition approaches, and the discussion of several implementations and Facial recognition criteria for preprocessing. In a holistic (geometric) approach the face image is viewed as a whole without searching for local characteristics. In the geometric method, recognition efficiency is greatly affected by illumination, orientation, and size. A commonly used computational technique for the identification of the face is a PCA (principal component analysis). The other commonly used method is eigenspace, which is a subspace defined by a set covariance matrix eigenvectors of image data. Linear discriminant analysis (LDA) provides the distinguishing within the class and between class objects.

A strategy involving Discrete Cosine Transform (DCT) has suggested by Hazim et. al. [3]. In this paper, at the decision and feature level, the local characteristics are retrieved and integrated.

Tudor et. al. [4] mentioned the technique of face recognition based on Gabor-filters. The article describes the k-neighborhood classifier for feature vectors and the supervised classification classifier. Wonjun et. al. [5] introduce a versatile facial recognition method for large-scale data sets collected during unregulated illumination variations. A new illumination insensitive method has been used in pre-processing, and linear discriminant analysis is used as a classifier for extracted Hybrid Fourier features.

Gabor filter is an appropriate model of image processing and one of the effective methods to explain the appearance of the face image. Feature Extraction is typically focused on the detailed portion of the face, including the parts of the mouth, eye, and eyebrow that are segmented from the illustration of the face [6]. The accuracy of the facial recognition system varies based on the choice of orientation and scale of the Gabor filters to extract the Gabor features. The pair of Gabor filters with various sizes and angles is implemented to the 
images to obtain face data. [7]. Therefore, the dimension and redundancy enlarged, the process of identification decelerated. Several methods are used to minimize dimensions and redundancy, without compromising the essential details on the face. [8]. In the proposed method, then Down-Sampling and Discrete Cosine Transform (DCT) eliminates unnecessary features and dimensions, and improves the accuracy of the system at a limited expense of calculations.

\section{RELATED WORK}

Extraction of features has been studied as the main step in face recognition techniques that are required to face identification.

The algorithm processed on the input image data of significant size, the significant size data must be relevant to the input image. The image also contains redundant data. A feature extraction technique is performed that can change the input image into a reduced feature set and decreased the redundant data.

\subsection{Gabor Filter As Feature Extraction Technique}

Mathematical description of the Gabor Kernel function in the next equation 2.1 [8].

$$
\Psi(\mathrm{x}, \mathrm{y}, \lambda, \theta)=\frac{1}{2 \Pi S \mathrm{xSy}} e^{-1 / 2\left(\frac{x^{\prime 2}}{S x * S x}+\frac{y^{\prime} 2}{S y * S y}\right)} e^{\mathrm{j} 2 \Pi \mathrm{x}^{\prime} / \lambda}
$$

The position of the pixels $(x, y)$ in the spatial domain, the wavelength $\lambda$ in pixels, Gabor filter angle $\theta$, and $S_{x}, S_{y}$ are the standard deviation in the particular directions $x$ and $y$ accordingly [9]. An equation follows the parameters $\mathrm{x}$ ' and $\mathrm{y}$ '.

$\mathrm{x}^{\prime}=\mathrm{x} \cos \theta+\mathrm{y} \sin \theta \quad \mathrm{y}^{\prime}=-\mathrm{x} \cos \theta+\mathrm{y} \sin \theta$

The Gabor features coefficients differentiated by the operation of the convolution operator between the input face image and the Gabor filter bank. The input facial picture $\mathrm{I}(\mathrm{x}, \mathrm{y})$ in the greyscale of the size $\mathrm{u}^{*} \mathrm{v}$ pixels as seen in equation 2.3.

$\mathrm{G}_{\mathrm{u}, \mathrm{v}}(\mathrm{x}, \mathrm{y})=\mathrm{I}(\mathrm{x}, \mathrm{y}) * \Psi(\mathrm{x}, \mathrm{y})$

The convolution outcomes (real and imaginary sections) of the face image reflect the powerful characteristics of spatial position, angle, and magnitude selectivity. These characteristics have extracted unique features, especially the eyes, nose, and mouth. Convolution outputs include distinct features of the interior, size, and angle; we combine exclusive features for a feature vector. [10].

Study Issues: - The Gabor filter is included in different sizes and different orientations to collect the features of the picture. When these operations run on the image, high dimensional and extensive redundant features are created. Certain filtering methods are used to minimize the high dimensions and the extensive redundancy of the features [11].

\subsection{Discrete Cosine Transform}

Discrete Cosine Transform is a linear frequency domain transform. DCT can express a finite sequence data that is a summation of different frequencies and magnitudes. The important characteristic of DCT is energy compaction, in that it's most energy or information is concentrated in its low-frequency elements [12].

The 2-D DCT of MxN matrix $\mathrm{A}$ has written as follows:

$$
B_{p Q}=a_{p} \alpha_{q} \sum_{m=0}^{M-1} \sum_{n=0}^{N-1} A_{m n} \cos \frac{\pi(2 m+1) p}{2 M} \cos \frac{\pi(2 n+1) q}{2 N}
$$

Where,

$$
\begin{gathered}
a_{p}= \begin{cases}1 / \sqrt{M}, & p=0 \\
\sqrt{2 / M}, & 1 \leq p \leq M-1\end{cases} \\
a_{q}= \begin{cases}1 / \sqrt{N}, & q=0 \\
\sqrt{2 / N}, & 1 \leq q \leq N-1\end{cases}
\end{gathered}
$$

and values of Bpq are the DCT coefficients of the A matrix [13].

DCT has transformed the initial signal into the frequency domain and the inverse DCT transform changed back in the initial signal from the transformed signal [12].

The large magnitude of the DCT coefficient is primarily located in the DCT matrix's top -left region. From the top left region, we can select the correct DCT coefficient and then convert it to a onedimensional vector that will be the classifier's input [8].

\subsection{Support Vector Machine}

The SVM is a supervised learning process with the intention of Classification of two-class problem. SVM selects a hyperplane with a maximum margin between two classes of training data, several hyperplanes can be drawn to differentiate the two classes of training data, and SVM selects the optimum hyperplane [14]. It is possible to describe the hyperplane as:

To $w x+b=0 \quad 2.5$

Where $\mathrm{x}$ is a hyperplane point, $\mathrm{w}$ represents the orthogonal to the hyperplane and $\mathrm{b}$ is the bias.

Multiple binary SVM has been combined for multiclass problems. In the proposed paper, ECOC (Error-correcting output code) is used for solving the multi-class problem [14] [15].

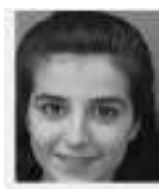

Fig. 1 ORL face image orientation

\section{PROPOSED WORK}

\subsection{Calculating Gabor Matrix}

The Gabor method projected upon this fig.1, ORL face database has a resolution of $112 \times 92$ pixel image, used five different angles and two different 
scaled, and gathered 10 Gabor matrices from every face image as shown in fig. 2 .

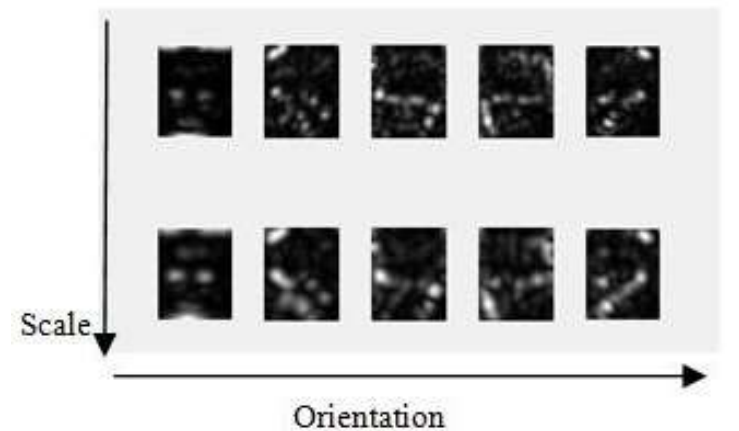

Fig. 2 Magnitude gabor filters of ORL face image.

The suggested Gabor Filter procedure, the downsampling of Gabor's feature coefficients along the row by factors two and along the column by factors five of all ten matrices, is evaluated and the Gabor sampled function vector is obtained. Then further reduction implementation by the DCT function.

\subsection{Performing DCT operation}

The DCT mapped the Gabor sampled features into a reduced feature space for the analysis in the frequency domain [14]. The DCT provides high compression with energy compaction [15] and DCT collected the low-frequency features from the sampled Gabor feature and would reshape it to a single-dimensional vector feature. This singledimensional feature vector is classified by the support vector machine.

\subsection{Training $S V M$}

The SVM classifier is trained by the feature vector of the training face image. In the training process, the labels also assign to the corresponding training image [16]. In the experiment, in every set of ORL face database, $80 \%$ of the image is used for the training image.

\subsection{ORL Image Dataset}

The ORL face dataset consists of images of 40 people as shown in fig. 3 , which is used for the face recognition system image data set. The pictures have been taken at various times, the contrast in lighting and the distinct facial expression, the images are taken from the frontal position of the people and others with a little side movement. The 8-bit grayscale images are accumulate in PGM format and the database consists of 40 folders and each folder has 10 images [14]. The total number of images is 400 .

\subsection{An Algorithm For The Execution Of Gabor -} DCT Feature Extraction Technique

Input: Grayscale Image

For loop scaling $=[0.5,1]$

Image_scaled $=$ RescaleImage;

For loop angle $=[0,45,90,135,180]$
Gabor [i] = Gabor_function (image_rescaled, angle)

End for

End for

Step 3: Down-sampling is applied along the row by factors two and along the column by factors in all ten Gabor's feature coefficient matrices.

Step 4: DCT is applied to down-sampled Gabor's feature coefficient matrix, and then the extracted low-frequency features are put in a feature vector. This feature vector is mapped by the SVM classifier.

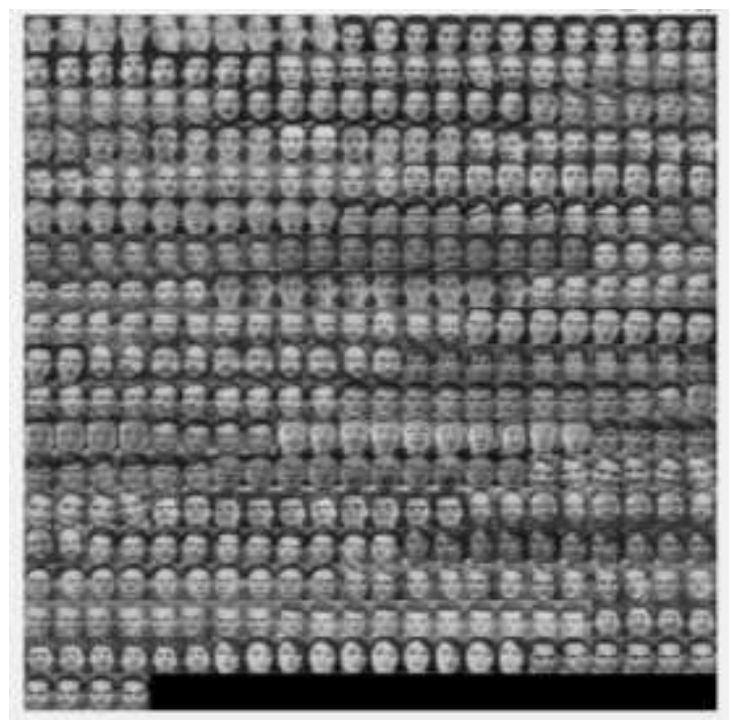

Fig. 3 All 400 ORL face image of 40 people

Table-4.1 Comparative Study of Gabor Sampling Filter and Suggested Gabor-DCT Filter

\begin{tabular}{|l|c|c|}
\hline Methods & Gabor Filter & $\begin{array}{c}\text { Proposed Gabor DCT } \\
\text { Filter Technique }\end{array}$ \\
\hline Accuracy (\%) & $\mathbf{7 0 . 8 6}$ & $\mathbf{9 6}$ \\
\hline
\end{tabular}

\section{EXPERIMENT AND RESULTS}

The proposed technique is performed in MATLAB $2018 \mathrm{~b}$ and the ORL dataset is used to perform the proposed methodology for the face recognition method. Training images are used in experiment 320 (80 percent) and 80 (20 percent) test images of 112 x 92 pixels in .pgm file format. The support vector machine classifier is used for classification. Crossvalidation calculates the accuracy of the system to retrieve the consistency of the output result. Next, the 5-fold cross-validation splits the trained model into 5 equal-sized subsets. It arbitrarily shuffles the images and divides the 400 images into 80 images of 5-block. The first 80 images are used as test data for the first run and the remaining 320 images are used for training. Similarly, the next 80 images are used for testing in the second run and the remaining ones will be used for training. It will work like this for 5 runs. The five-fold cross-validation estimates the average accuracy of almost 96 percent as shows in table-1. 


\section{CONCLUSION}

This paper analyzed Gabor Filter, Sampling, Discrete Cosine Transform, Support Vector Machine, and 5-fold Cross-validation for Face Recognition system in different expressions and illumination conditions. Gabor features are extremely dimensional and massive redundant features. Calculation with all of these features was lengthy and high-cost calculation; this is the key issue in Gabor filter extraction. Several filtering methods exist, which can convert the high dimension into low dimension and have reduced redundancy features. Then, in the presented extraction method, the Gabor features were filtered by the sampling used, and then the Gabor sampled features were processed by the DCT. Ultimate vector feature designed with a low-frequency extracted feature. The experimental result of the research showed that the suggested hybrid approach has an accuracy of 96 percent. The experimental result shows that the proposed approach has better accuracy than the existing conventional Gabor filter approaches for various expressions and illumination conditions.

\section{REFERENCES}

[1] Z.M. Hafed and M.D Levine. Face recognition using discrete cosine transform. Journal of Computer Vision, 43(3):167-188, 2001

[2] Zhao. W., Chellappa. R., Phillips. P.J. and Rosenfeld. A. (2003) 'Face Recognition: A Literature Survey' Journal of ACM Computing Surveys (CSUR), Vol. 35, No. 4, December, pp. 399- 458.

[3] Hazim. K.E. and Rainer.S. (2006) 'Local Appearance based Face Recognition Using Discrete Cosine Transform' 13th European Signal Processing Conference (EUSIPCO).

[4] Tudor. B. (2010) 'Gabor or Filter-based Face Recognition Technique' Proceeding of The Romanian Academy, Series A, Vol.11, No. 3, pp. 277-283.

[5] W. Hwang, H. Wang, H. Kim, S. Kee and J. Kim, "Face Recognition System Using Multiple Face Model of Hybrid Fourier Feature Under Uncontrolled Illumination Variation," in IEEE Transactions on Image Processing, vol. 20, no. 4, pp. 1152-1165, April 2011, doi: 10.1109/TIP.2010.2083674.

[6] WeiFeng Liu and ZengFu Wang. Face recognition based on fusion of multiple Gabor features. In IEEE International conference on Pattern Recognition.IEEE, 2006.

[7] Fenguin Chen Zhiliang Wang Zhengguang Xu Jianng Xiao and Guojiang Wang. Face recognition using wavelet transform and neural network ensemble. In Internation Symposium on Intelligent Information Technology Application, volume 24, pages 871-875. IEEE, 2008.

[8] Meihua Wang, Hong Jiang and Ying Li, "Face recognition based on DWT/DCT and SVM," 2010 International Conference on Computer Application and System Modeling (ICCASM 2010), Taiyuan, 2010, pp. V3-507-V3-510.

[9] Xiaoli Li Qiuqi Ruan Chengxiong Ruan, "Facial Expression Recognition with Local GaborFilters", International conference, IEEE, 2010.

[10] Guoqiang Wang and Zongying Ou, "Face Recognition Based on Image Enhancement and Gabor Features," 2006 6th World Congress on Intelligent Control and Automation, Dalian, 2006, pp. 9761-9764, doi: 10.1109/WCICA.2006.1713900.

[11] S. Nazari, M.S. Moin, "Face recognition and local Gabor features", in international conference on electrical engineering (ICEE), pp 1-4, IEEE, 2013.

[12] S. Ajitha, A. Annis Fathima, V. Vaidehi, M. Hemalatha and R. Karthigaiveni, "Face recognition system using Combined Gabor Wavelet and DCT approach," 2014 International Conference on Recent Trends in Information Technology, Chennai, 2014, pp. 1-6, doi: 10.1109/ICRTIT.2014.6996156.

[13] https://www.mathworks.com/help/images/discrete-cosinetransform.html

[14] S. Nasr, K. Bouallegue, M. Shoaib and H. Mekki, "Face recognition system using bag of features and multi-class SVM for robot applications," 2017 International Conference on Control, Automation and Diagnosis (ICCAD), Hammamet, 2017, pp. 263-268, doi: 10.1109/CADIAG.2017.8075668.

[15] S. M. Jaisakthi and C. Aravindan, "Face detection based on eigenfaces and legendre moments," TENCON 2009 - 2009 IEEE Region 10 Conference, Singapore, 2009, pp. 1-5, doi: 10.1109/TENCON.2009.5396153. 